\title{
Electron Wave Function at a Vicinal Surface: Switch from Terrace to Step Modulation
}

\author{
J.E. Ortega, ${ }^{1}$ S. Speller, ${ }^{2}$ A. R. Bachmann, ${ }^{2}$ A. Mascaraque,${ }^{3}$ E. G. Michel,${ }^{3}$ A. Närmann, ${ }^{1}$ A. Mugarza,${ }^{1}$ \\ A. Rubio, ${ }^{1, *}$ and F. J. Himpsel ${ }^{4}$ \\ ${ }^{1}$ Centro Mixto CSIC-UPV and Donostia International Physics Center, Departamento de Física Aplicada I, \\ Universidad del País Vasco, Plaza Oñate 2, 20018-San Sebastián, Spain \\ ${ }^{2}$ Fachbereich Physik, Universität Osnabrück, 49069 Osnabrück, Germany \\ ${ }^{3}$ Departamento de Física de la Materia Condensada, Universidad Autónoma de Madrid, E-28049 Madrid, Spain \\ ${ }^{4}$ Physics Department, University of Wisconsin Madison, 1150 University Avenue, Madison, Wisconsin 53706-1390
}

(Received 2 November 1999)

\begin{abstract}
The $\mathrm{Cu}(111)$ surface state has been mapped for vicinal surfaces with variable step densities by angleresolved photoemission. Using tunable synchrotron radiation to vary the $k$ dependence perpendicular to the surface, as well as the $k_{\|}$dependence, we find a switch between two qualitatively different regimes at a miscut of $7^{\circ}$ (17 $\AA$ terrace width). For larger miscut angles the step modulation of the wave function dominates, and for smaller miscut angles the terrace modulation dominates. These observations resolve an apparent inconsistency between prior photoemission and STM results.
\end{abstract}

PACS numbers: 79.60.Bm, 73.20.Dx

Vicinal or faceted surfaces play an important role in producing low-dimensional, nanostructured solids with tailored electronic properties [1-3]. They have also served as models for the special sites which dominate the heterogeneous catalysis [4]. In both situations the basic issue at hand is the nature of the wave function that tailors the electronic states and characterizes the chemically active orbitals. The wave function of a step lattice is modulated by two periodicities, that of the superlattice [Fig. 1(a)] and that of the atomic corrugation of the terraces [Fig. 1(b)]. In addition, as we will show, the modulation by the bulk lattice perpendicular to the surface plays an important role. A good model system for investigating the behavior of these wave functions is a noble metal surface with a free-electron-like surface state, such as $\mathrm{Cu}(111)$ [5-10]. It has been shown that regular superlattices of straight, monoatomic steps can be prepared on vicinal $\mathrm{Cu}(111)$ at $300 \mathrm{~K} \mathrm{[10].} \mathrm{The} \mathrm{Cu}(111)$ surface state lies close to the Fermi level. Its charge density produces well-defined ripples in scanning tunneling microscopy (STM) pictures [5-7]. These ripples lie in the (111) plane of the terraces, thus giving rise to a model wave function similar to that in Fig. 1(b). Angle-resolved photoemission data, on the other hand, produce $E(k)$ band dispersions that lead to the picture in Fig. 1(a) [8-10]. In addition, backfolding of the surface state band by the reciprocal vector of the step superlattice has been reported and explained in a model consistent with Fig. 1(a) [9].

In order to find out whether or not these two models are compatible with each other we have performed angleresolved, photon-energy-dependent photoemission studies at $\mathrm{Cu}(111)$ surfaces with various miscut angles. For large miscuts $\left(9^{\circ}\right)$ the surface band dispersions reproduce previous photoemission data, but for small miscuts $\left(5^{\circ}\right)$ they are centered about the terrace normal as found in STM measurements [7]. The terrace width where this switch occurs (17 $\AA$ or eight atomic rows) defines a characteristic length scale, where the description of the surface wave function changes qualitatively, from being modulated by the step lattice to being modulated by the terrace corrugation. This length scale is shorter than the electron coherence length on flat terraces, but very similar to the minimum wave vector that couples surface states with bulk states. Our quantitative analysis uses a picture based on electron diffraction at step lattices [1]. It includes the momentum perpendicular to the surface, which is varied by changing the kinetic energy of the photoelectrons via the photon energy.

Vicinal $\mathrm{Cu}(111)$ surfaces were prepared with miscuts between $5^{\circ}$ and $9^{\circ}$ towards the $[1 \overline{1} 2]$ azimuthal direction. The crystals were electrochemically polished, sputter-annealed $\left(500 \mathrm{eV} \mathrm{Ar}^{+}\right.$ions, $\left.800 \mathrm{~K}\right)$, and cooled slowly to $300 \mathrm{~K}$. STM shows a regular distribution of monoatomic, (100)like steps running along the [110] direction. We have chosen surfaces with (100)-like steps instead of (111)-like, since regular arrays are easier to prepare. Photoemission experiments were performed using $p$-polarized synchrotron radiation using a vacuum generator analyzer at HASYLAB and a Scienta analyzer at the SRC (Wisconsin). The measurement geometry is displayed in Fig. 1. The terrace width $d$ varies from 13.3 to $23.9 \AA$ for $\alpha=9^{\circ}$
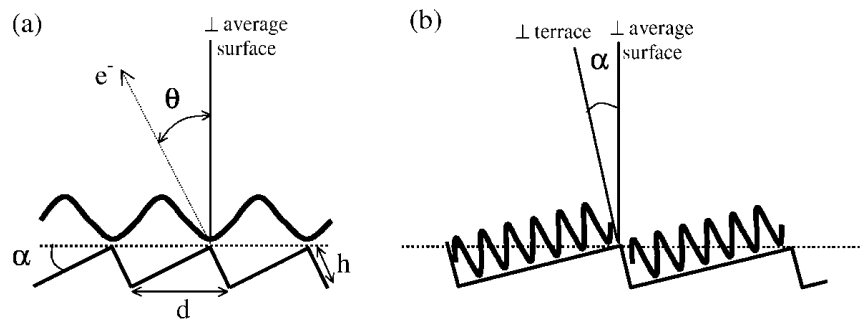

FIG. 1. Schematic description of the two different models for the wave function on vicinal surfaces. The measurement geometry for angle-resolved photoemission experiments is included. 
and $\alpha=5^{\circ}$ miscuts, respectively. Emission angles $(\theta)$ are given with respect to the normal of the average surface.

In Fig. 2 we present the dispersion of the $p_{z}$-like surface state band as a function of $\theta$ in the direction perpendicular to the steps, measured at three different photon energies. High photoemission intensity is shown in the dark areas. Parabolic fits (thick lines) show that the bottom of the band is shifted up from the value of the flat (111) surface $(-0.4 \mathrm{eV}$ [11]) to -0.35 and $-0.3 \mathrm{eV}$ for $5^{\circ}$ and $9^{\circ}$ miscut, reflecting the repulsive effect of the step barrier [10]. But the most remarkable feature, compared to flat $\mathrm{Cu}(111)$, is the systematic shift of the band minimum $\left(\theta^{\mathrm{min}}\right)$ away from normal emission. It lies at $12^{\circ}, 7.6^{\circ}$, and $4.8^{\circ}$ for 10,13 , and $22 \mathrm{eV}$, respectively, in the $9^{\circ}$ miscut surface. For the $5^{\circ}$ miscut it is located at $9.3^{\circ}, 7.9^{\circ}$, and $6.2^{\circ}$, respectively [12]. For flat $\mathrm{Cu}(111)$ the surface band is centered around $0^{\circ}$ at all photon energies. Since the component of the electron wave vector parallel to the average surface $\left(k_{\|}\right)$is conserved during the photoemission process, these results indicate a nonzero value $k_{\|}^{\min }$ at the bottom of the band for the vicinal surfaces. The numerical values of $k_{\|}^{\min }$ can be obtained from the

(a)

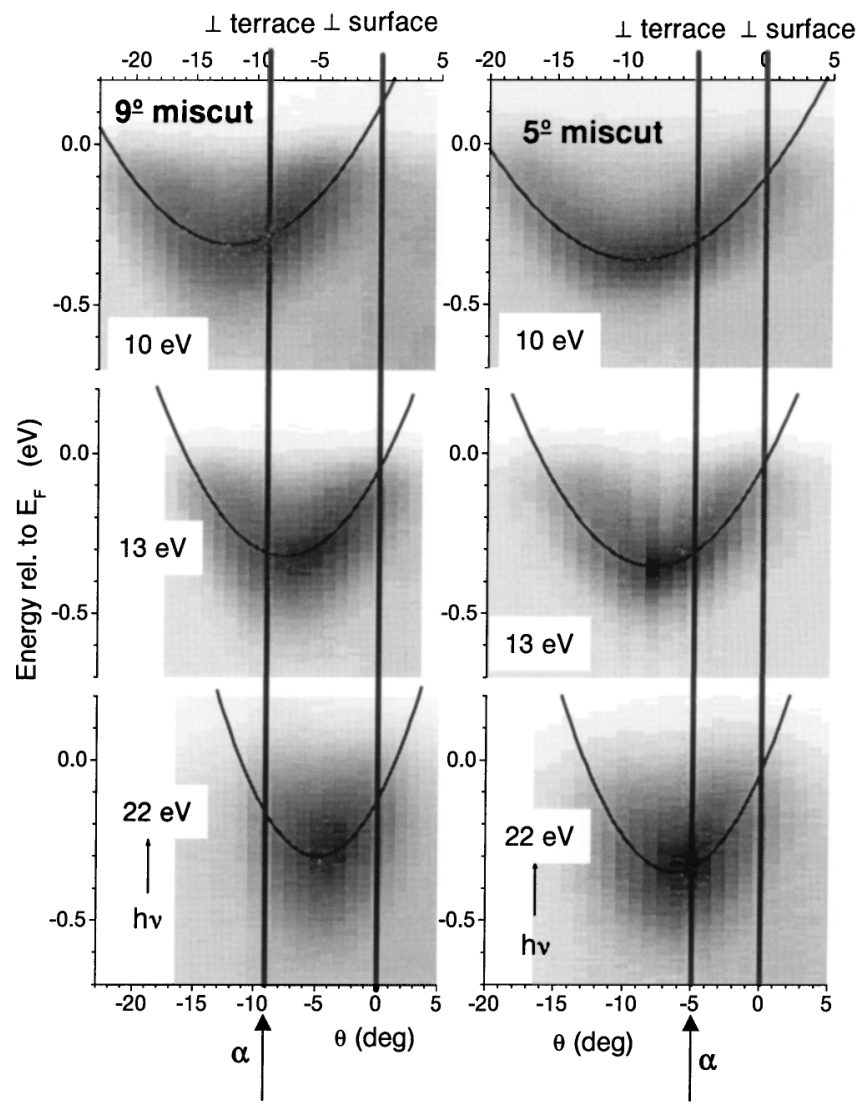

FIG. 2. Photoemission intensity (dark) versus energy and emission angle taken with different photon energies showing the $p_{z}$-like surface band for stepped $\mathrm{Cu}(111)$ surfaces with (a) $9^{\circ}$ and (b) $5^{\circ}$ miscut angles. measured kinetic energy $E_{\mathrm{kin}}(\theta)$ assuming energy and $k_{\|}$ conservation:

$$
k_{\|}=\sqrt{\left(2 m / \hbar^{2}\right) E_{\mathrm{kin}}} \sin \theta .
$$

Note that, as a consequence of this relation, a fixed $k_{\|}^{\min }$ gives rise to a $\theta^{\mathrm{min}}$ that varies with $E_{\mathrm{kin}}$ and approaches zero at the high kinetic energies obtained for high photon energies $(h \nu)$. This is observed in Fig. 2 for the $9^{\circ}$ miscut. The emission angles in this case are not correlated with the terrace normal $(\alpha)$, since $\theta^{\min }>\alpha$ for small $h \nu$ and $\theta^{\min }<\alpha$ for large $h \nu$. The $5^{\circ}$ sample behaves qualitatively different from the $9^{\circ}$ sample. The emission angle $\theta^{\mathrm{min}}$ does not approach the average surface normal $\theta=0^{\circ}$, but instead approaches the terrace normal $\theta^{\min }=\alpha$. In order to understand this result, as we will show, we need to invoke the perpendicular wave vector $k_{\perp}$.

In Fig. 3 we analyze the photoemission experiment using the basic framework of low energy electron diffraction from a vicinal surface [1]. The diffraction rods are defined by multiples of the step superlattice vector $G_{x}=2 \pi / d$. Note the change in the vertical scale for the $5^{\circ}$ miscut. The data points (dots) represent the different (a)
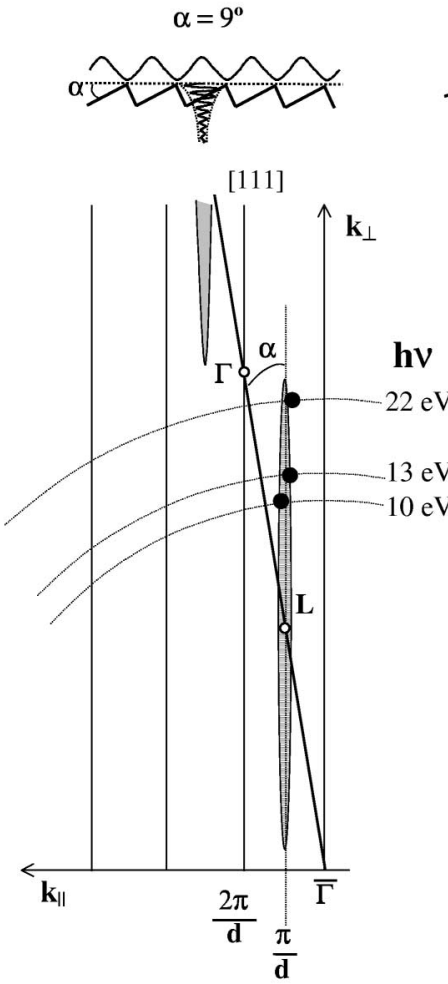

(b)
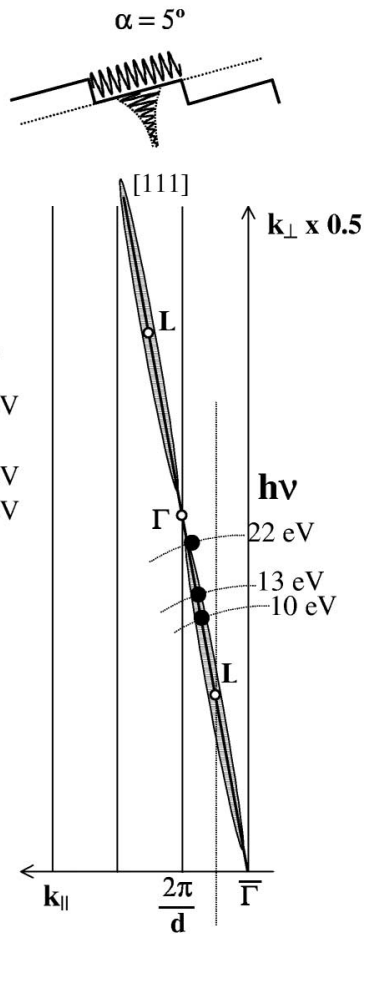

FIG. 3. Description of the photoemission experiment from a vicinal surface using an electron diffraction model. The dots are three-dimensional $\mathbf{k}$ values obtained from the data in Fig. 2 via Eqs. (1) and (3). The cigar-shaped shaded hatched regions represent the spread of the surface state wave function in $\mathbf{k}$ space. The corresponding wave function in real space is indicated on top. A characteristic switch of the orientation occurs at a critical miscut angle of about $7^{\circ}$. 
$k_{\|}^{\text {min }}$ calculated from Eq. (1). For a two-dimensional or a one-dimensional state the value of $k_{\|}^{\min }$ is a good quantum number and should be independent of measurement parameters, such as the photon energy. This is indeed the case for the $9^{\circ}$ miscut since the dots line up at $k_{\|}^{\min }=\pi / d$. For the $5^{\circ}$ sample, on the other hand, $k_{\|}^{\min }$ varies with photon energy. Consequently, we have to bring in the perpendicular momentum $k_{\perp}$. To a first approximation, $k_{\perp}$ can be obtained from a parabolic, freeelectron-like band in a constant inner potential $V_{0}[13,14]$ :

$$
\begin{gathered}
E_{\mathrm{kin}}(\mathbf{k})=\hbar^{2} \mathbf{k}^{2} / 2 m-V_{0} \quad\left(V_{0}>0\right), \\
k_{\perp}=\sqrt{\left(2 m / \hbar^{2}\right)\left(E_{\mathrm{kin}}+V_{0}\right)-k_{\|}^{2}},
\end{gathered}
$$

where $\mathbf{k}$ is the photoelectron wave vector inside the crystal, $E_{\text {kin }}(\mathbf{k})$ is its energy referenced to the vacuum level, and $V_{0}$ represents the potential step at the surface $\left(V_{0}=\right.$ $13.5 \mathrm{eV}$, following Ref. [14]). At a fixed photon energy $h \nu$ the kinetic energy is fixed as well, giving rise to the circles defining the position of the final state electron in momentum space, as shown in Fig. 3. The experimental data points (dots) are determined by the intersection of these circles with the $k_{\|}$value obtained from Eq. (1). We now find that the data points for the $5^{\circ}$ sample line up along the terrace normal in three-dimensional $\mathbf{k}$ space.

After characterizing the location of the surface state in $\mathbf{k}$ space we are now able to draw conclusions about the nature of its wave function. The two extreme cases discussed earlier in Figs. 1(a) and 1(b) are shown on top of Fig. 3. The wave function perpendicular to the surface has been added, in order to explain transitions in three-dimensional $\mathbf{k}$ space. For a true, two-dimensional surface state, the wave function decays perpendicular to the surface giving rise to a broadened $k$ distribution in the direction perpendicular to the average surface $\left(k_{\perp}\right)$ [15]. That is indicated by the cigar-shaped shaded hatched areas in Fig. 3(a). For the $p_{z}$-like surface state in $\mathrm{Cu}(111)$, the $k$ distribution is centered at the $L$ point of the bulk band structure $\left[\mathbf{k}_{L}=(1 / 2,1 / 2,1 / 2) 2 \pi / a\right]$, where $a$ is the $\mathrm{Cu}$ lattice constant. As a consequence, the photoemission intensity exhibits a maximum when the perpendicular momentum of the final state lies close to $L$ [16].

For the case of low miscut angles shown in Fig. 3(b) we find empirically that the $k$ distribution of the surface state is confined to cigar-shaped areas that are lined up parallel to the terrace normal. From that, we conclude that the wave function decays perpendicular to the terraces, not perpendicular to the average surface. It appears as if the wave function ignored the step lattice and propagated parallel to the (111) terraces. Such behavior is seen in STM. In fact, previous STM work was performed with small miscut angles $\left(<5^{\circ}\right)$ or near isolated steps [5-7]. Thus, we are able to reduce the apparent discrepancy between previous photoemission and STM data to the fact that photoemission was performed at large miscuts $\left(>8^{\circ},[8,9]\right)$, whereas
STM covered only small miscuts. By covering the whole range of miscuts in one photoemission experiment we are able to follow the switch between the two regimes [17].

How can the switch between the two types of wave functions in Figs. 1(a) and 1(b) be explained? A straightforward explanation of the switch might be based on the lateral coherence length of the electrons. If the terrace width becomes large compared to the coherence length, the electron wave function of an electron placed at the center of a terrace does not see the step edges. The problem with such a simple explanation is that it requires a lateral coherence length that is much smaller than expected from transport measurements (about $300 \AA$ ). Previous photoemission experiments $[11,18]$ gave a significantly smaller coherence length of $30 \AA$ but still larger than the $17 \AA$ terrace width for a $7^{\circ}$ miscut where the switch occurs. Unless there is an extra elastic scattering mechanism at stepped surfaces [11], this model cannot explain the switch.

A second mechanism is coupling between surface and bulk states via step lattice vectors $G_{x}=2 \pi / d$. Such a process has been pointed out by Hörmandinger and Pendry for rows of scatterers on flat $\mathrm{Cu}(111)$ [19]. Figure 4 outlines schematically Bragg scattering with $G_{x}$ vectors from surface states on the terrace, at the bottom of the surface band $\left(k_{\|}=0\right)$. Note that the cigars in Fig. 3 describe the Fourier spectrum of a decaying wave with $\mathbf{k}=\mathbf{k}_{L}=$ $(1 / 2,1 / 2,1 / 2)(2 \pi / a)$. When the step spacing $d$ becomes small enough, bulk states at the edge of the band gap can be reached by adding $G_{x}$. In such case bulk states hybridize with the surface state wave function giving rise to a three-dimensional surface resonance. In Fig. 4 the thick line represents the constant energy line for bulk states at about $-0.3 \mathrm{eV}$. Since the band edge is very steep, the constant energy surface at $-0.3 \mathrm{eV}$ is very close to the Fermi surface, such that the minimum angle for coupling is approximately given by $\cos \alpha=k_{F \text {,neck }} / G_{x}$, i.e., $\sin 2 \alpha=\left(k_{F, \text { neck }} a\right) /(\pi \sqrt{3})$. Taking $k_{F, \text { neck }}=0.26 \AA^{-1}$

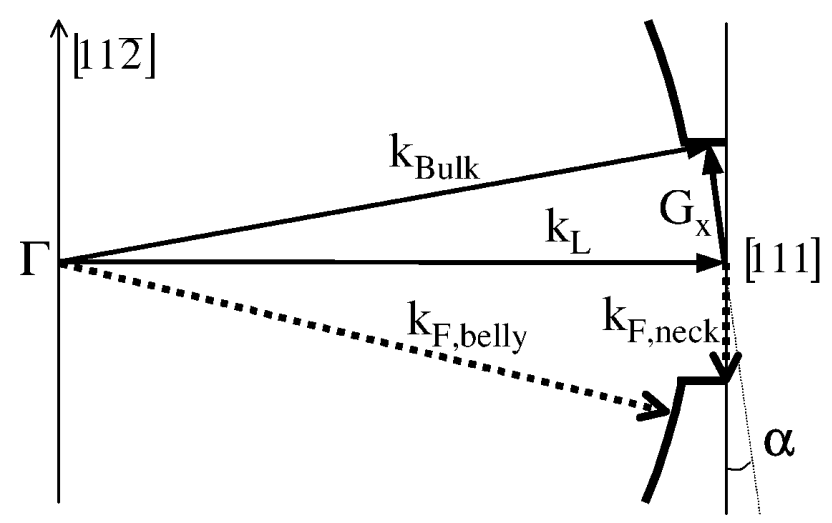

FIG. 4. Energy-conserving scattering from surface states to bulk states via $G_{x}$ superlattice vectors. The thick line represents roughly a constant energy line at the edge of the band gap close to $E_{F}$. The minimum angle to reach this line is given approximately by $\cos \alpha=k_{F, \text { neck }} / G_{x}$. 
from Ref. [20], we obtain $\alpha \approx 5^{\circ}$. Thus, for miscut angles above $5^{\circ}$, surface states become resonances. Our data indicate that these surface resonances lie on the average surface plane.

Finally, to explain the critical angle of the switch-over we can also consider the width of the gap in the projected band structure that supports the $\mathrm{Cu}(111)$ (terrace) surface state. It is located in the $L$ neck of the $\mathrm{Cu}$ Fermi surface. Considering bulk states in the first Brillouin zone, this gap shrinks to zero at a miscut angle between $7.2^{\circ}$ and $9.1^{\circ}$ for energies between $-0.35 \mathrm{eV}$ and $E_{F}$. Like surface-bulk coupling, this mechanism describes again the conversion of a surface state into a resonance. Based on our data, it actually gives the correct range for the critical miscut angle. Thus we may consider such a gap closing as the mechanism that determines the switch-over between average surface and terracelike wave functions.

J.E. O. and A. Mu. are supported by the Universidad del País Vasco (UPV057.240-EA197/97 and 026/98)and the Max Planck Research Award Program. E. G. M. and A. Ma. are funded by the Spanish DGES (PB-970031) and the Comunidad de Madrid (07N/0031/1998). Experiments were performed at Hasylab within the TMR Contract ERBFMGECTP950059 and at the SRC, which is supported by the NSF under Award No. DMR-9531009. F. J. H. is funded by NSF DMR-9815416. Helpful discussions with P. M. Echenique, F. J. García de Abajo, and E. V. Chulkov are acknowledged.

* On leave from Departamento de Física Teórica, Universidad de Valladolid, E-47011, Valladolid, Spain.

[1] M. Henzler, Appl. Phys. A 9, 11 (1976).

[2] E. D. Williams and N. C. Bartelt, Science 251, 393 (1991); Z. Toroczkai and E. D. Williams, Phys. Today 52, No. 12, 24 (1999); J.E. Ortega, F. J. Himpsel, R. Haight, and D. R. Peale, Phys. Rev. B 49, 13859 (1994); H. Namba, N. Nakanishi, T. Yamaguchi, and H. Kuroda, Phys. Rev. Lett. 71, 4027 (1993).

[3] R. Nötzel and K.H. Ploog, Adv. Mater. 5, 22 (1993); R. Nötzel, Z. Niu, M. Ramsteimer, H.P. Schönherr, A. Trampert, L. Däweritz, and K.H. Ploog, Nature (London) 392, 56 (1998); F. J. Himpsel, J. E. Ortega,
G. J. Mankey, and R. F. Willis, Adv. Phys. 47, 511 (1998); P. Segovia, D. Purdie, M. Hegsberger, and Y. Baer, Nature (London) 402, 504 (1999).

[4] M. B. Salmeron and G. A. Somorjai, Surf. Sci. 90, 373 (1980); T. Zambelli, J. Wintterlin, J. Trost, and G. Ertl, Science 273, 1688 (1996); C. E. Tripa and J. T. Yates, Jr., Nature (London) 398, 591 (1999).

[5] M.F. Crommie, C.P. Lutz, and D.M. Eigler, Nature (London) 363, 524 (1993); 369, 464 (1994).

[6] Y. Hasegawa and Ph. Avouris, Phys. Rev. Lett. 71, 1071 (1993); Ph. Avouris and I.-W. Lyo, Science 264, 942 (1994); Ph. Avouris, I.-W. Lyo, and R. E. Walkup, J. Vac. Sci. Technol. B 12, 1447 (1994).

[7] L. Bürgi, O. Jeandupeux, A. Hirstein, H. Brune, and K. Kern, Phys. Rev. Lett. 81, 5370 (1998); O. Jeandupeux, L. Bürgi, A. Hirstein, H. Brune, and K. Kern, Phys. Rev. B 59, 15926 (1999).

[8] A. P. Shapiro, T. Miller, and T.-C. Chiang, Phys. Rev. B 38, 1779 (1988).

[9] X. Y. Wang, X. J. Shen, and R. M. Osgood, Jr., Phys. Rev. B 56, 7665 (1997).

[10] O. Sánchez, J. M. García, P. Segovia, J. Alvarez, A. L. Vázquez de Parga, J.E. Ortega, M. Prietsch, and R. Miranda, Phys. Rev. B 52, 7894 (1995).

[11] J. Tersoff and S. D. Kevan, Phys. Rev. B 28, 4267 (1983).

[12] Since the sample is not moved during data acquisition, the biggest uncertainty $\left( \pm 0.5^{\circ}\right)$ affects the relative position of terrace and average normal with respect to the three parabola.

[13] F. J. Himpsel, Adv. Phys. 32, 1 (1983).

[14] P. Thiry, D. Chandesris, J. Lecante, C. Guillot, R. Pinchaux, and Y. Petroff, Phys. Rev. Lett. 43, 82 (1979).

[15] S. D. Kevan, N. G. Stoffel, and N. V. Smith, Phys. Rev. B 31, 1788 (1985); 31, 3348 (1985).

[16] S. G. Louie, P. Thiry, R. Pinchaux, Y. Petroff, D. Chandesris, and J. Lecante, Phys. Rev. Lett. 44, 549 (1980).

[17] More recent data taken at an intermediate miscut angle of $7.3^{\circ}$ show data points lying in between the [111] and the average surface direction. They will be published elsewhere.

[18] F. Theilmann, R. Matzdorf, G. Meister, and A. Goldmann, Phys. Rev. B 56, 3632 (1997); S. D. Kevan, Phys. Rev. Lett. 50, 526 (1983).

[19] G. Hörmandinger and J. B. Pendry, Phys. Rev. B 50, 18607 (1994).

[20] P. T. Coleridge and I. M. Templeton, Phys. Rev. B 25, 7818 (1982). 